\title{
Criminals Treated as Predators to Be Harvested: A Two Prey One Predator Model with Group Defense, Prey Migration and Switching
}

\author{
J. Sooknanan ${ }^{1}$, B. Bhatt ${ }^{1} \&$ D. M. G. Comissiong ${ }^{1}$ \\ ${ }^{1}$ Department of Mathematics and Statistics, The University of the West Indies, St. Augustine, Trinidad and Tobago \\ Correspondence: D. M. G. Comissiong, Department of Mathematics and Statistics, The University of the West In- \\ dies, St. Augustine, Trinidad and Tobago. Tel: 1-868-662-2002 Ext. 83099. E-mail: \\ Donna.Comissiong@sta.uwi.edu
}

Received: June 5, 2012 Accepted: July 8, 2012 Online Published: July 20, 2012

doi:10.5539/jmr.v4n4p92 URL: http://dx.doi.org/10.5539/jmr.v4n4p92

\begin{abstract}
A mathematical model is formulated and analysed to study the dynamics of a one predator - two prey eco-system using the logistic equation in which the predator switches between habitats and is being harvested. Constant effort and Constant yield harvesting functions are applied to the predators. The Constant effort strategy as opposed constant yield strategy may lead to an additional equilibrium in which the predators are extinct, whereas in the Constant yield stategy the only equilibrium is the coexistence one. In both cases, computer simulations are performed to study the effects of various parameters on the dynamics of the system. These studies show that depending on parameter values, a stable equilibrium or a limit cycle orbit is possible with both harvesing types.
\end{abstract}

Keywords: predator prey model, switching, group defense, harvesting

\section{Introduction}

When choosing a victim, criminals like predators in nature will seek out the more vulnerable prey. Prey in response to this threat may organise themselves in groups to protect themselves. This is known as group defense and may result in the predator switching to a habitat with fewer prey. Similarly, group defense in the form of neighborhood watches and cooperation may be a strong deterrent to criminals, and result in the movement of criminals from one area to another. This switching of territory or habitat is known as spatial crime displacement (Eck, 1993). Concomitant with crime is its nemesis the police, who remove or harvest the criminals from the system. In mathematical ecology, there are two standard harvesting strategies (Brauer \& Castillo-Chavez, 2001) - constant effort harvesting and constant yield harvesting. In proportional or constant effort harvesting, a fixed proportion of the predators are removed. In comparison, in constant yield or constant - rate harvesting a constant number of individuals per unit of time are removed from the system. In this research we examine both strategies of crime control. Our goals in this paper are to explore the dynamics of such a predator - prey ecosystem, and to determine how the different harvesting policies affect the existence and stability properties of equilibria and periodic solutions.

Models of this form have been studied (Khan et al.,1998 \& 2004; Bhatt et al., 2005; Bhattacharyya \& Mukhopadhyay, 2006). Khan et al. (2004) and Bhatt et al. (2005) both considered a two-prey one-predator model with migration, switching and prey group defence. Whilst Bhatt et al. (2005) considered a prey growth rate proportional to the number of prey, Khan et al. (2004) considered logistic growth in the prey. This resulted in a hopf bifurcation for switching index $n=1$ for certain parameter values. Bhattacharyya \& Mukhopadhyay (2006) considered a similar model to (Khan et al., 2004) for the switching indexes $n=1$ and $n=2$ with and without prey migration. However, unlike switching models, he modelled migration of the prey population between the habitats through the process of physical diffusion by spatially extending the models. We consider a general form of this model as well as constant yield and constant effort harvesting of the predators. Khan et al. (2004) also considered a bifurcation analysis using the carrying capacity $k$ as the bifurcation parameter, whereas our emphasis is on the harvesting terms which are analogous to the removal of criminals by the police.

The paper is organized as follows: Section 2 contains a description of the model. The different equilibria and their stability for constant effort harvesting and constant yield harvesting are analysed in Section 3 and Section 4. 
Section 5 presents the discussion and the conclusion is given in Section 7 .

\section{The Model}

A one-predator two-prey model is considered. The prey $V_{1}$ and $V_{2}$ are assumed to grow with a logistic growth rate since there are limits to growth, such as finite space or food supply and each population competes with itself for resources. However, the two prey species do not compete against each other. The population dynamics of the predator $y$ are dependent on the conversion of prey to predator from the foraged prey $\left(\alpha_{1}, \alpha_{2}\right)$ and the harvesting function $h(y)$. The prey may migrate between the two areas with a probability $\epsilon_{1} p_{12}$ and $\epsilon_{2} p_{21}$. The parameters are described in Table 1.

Table 1. Parameters used in the switching model

\begin{tabular}{ll}
\hline Parameter & Description \\
\hline$r_{1}, r_{2}$ & intrinsic growth rates of prey species inside the two areas \\
$K_{1}, K_{2}$ & carrying capacities of the prey species inside the two areas \\
$\epsilon_{i}$ & Normal migration rates inversion barrier strength in going out of \\
& habitat $\epsilon_{i}$ - from one area to the other \\
$p_{i j}$ & the probability of successful transition from the ith to the jth area \\
$\epsilon_{i} p_{i j}$ & probability of migrating from the ith to the jth area \\
$m$ & constant such that $0 \leqslant m \leqslant 1$ \\
& $m=0 \Rightarrow$ no attacked prey leaves the system \\
$\beta_{1}, \beta_{2}$ & $m=1 \Rightarrow$ all those attacked leave the system \\
$\alpha_{1}, \alpha_{2}$ & the predator response rate towards the prey - the hunting rates \\
$h(y)$ & harvesting function - removal of criminals by police \\
$n$ & switching index
\end{tabular}

We consider the general model:

$$
\begin{gathered}
V_{1}^{\prime}=r_{1} V_{1}\left(1-\frac{V_{1}}{K_{1}}\right)+\epsilon_{2} p_{21} V_{2}-\epsilon_{1} V_{1}-\frac{m \beta_{1} V_{1} y}{1+\left(\frac{V_{1}}{V_{2}}\right)^{n}}, \\
V_{2}^{\prime}=r_{2} V_{2}\left(1-\frac{V_{2}}{K_{2}}\right)+\epsilon_{1} p_{12} V_{1}-\epsilon_{2} V_{2}-\frac{m \beta_{2} V_{2} y}{1+\left(\frac{V_{2}}{V_{1}}\right)^{n}}, \\
y^{\prime}=\frac{\alpha_{1} \beta_{1} V_{1} y}{1+\left(\frac{V_{1}}{V_{2}}\right)^{n}}+\frac{\alpha_{2} \beta_{2} V_{2} y}{1+\left(\frac{V_{2}}{V_{1}}\right)^{n}}-h(y) y, \\
V_{1}, V_{2}, \quad y \geqslant 0 .
\end{gathered}
$$

For constant effort harvesting $h(y)=h$. Here $h y$ in Equation (3) is the harvesting yield per unit time with $h$ a measure of the effort expended in harvesting. For $n=1$, the system becomes:

$$
\begin{gathered}
V_{1}^{\prime}=r_{1} V_{1}\left(1-\frac{V_{1}}{K_{1}}\right)+\epsilon_{2} p_{21} V_{2}-\epsilon_{1} V_{1}-\frac{m \beta_{1} V_{1} V_{2} y}{V_{1}+V_{2}}, \\
V_{2}^{\prime}=r_{2} V_{2}\left(1-\frac{V_{2}}{K_{2}}\right)+\epsilon_{1} p_{12} V_{1}-\epsilon_{2} V_{2}-\frac{m \beta_{2} V_{1} V_{2} y}{V_{1}+V_{2}}, \\
y^{\prime}=\frac{\alpha_{1} \beta_{1} V_{1} V_{2} y}{V_{1}+V_{2}}+\frac{\alpha_{2} \beta_{2} V_{1} V_{2} y}{V_{1}+V_{2}}-h y .
\end{gathered}
$$

In the harvesting industry, constant effort is not the only method of harvesting. Another way of harvesting is removal at the constant rate $H$ per unit time known as constant rate or constant yield harvesting. For $n=1$, the system of Equations (1) to (3) becomes:

$$
V_{1}^{\prime}=\left(r_{1}-\epsilon_{1}\right) V_{1}-\frac{r_{1} V_{1}^{2}}{K_{1}}+\epsilon_{2} p_{21} V_{2}-\frac{m \beta_{1} V_{1} V_{2} y}{V_{1}+V_{2}},
$$




$$
\begin{gathered}
V_{2}^{\prime}=\left(r_{2}-\epsilon_{2}\right) V_{2}-\frac{r_{2} V_{2}^{2}}{K_{2}}+\epsilon_{1} p_{12} V_{1}-\frac{m \beta_{2} V_{1} V_{2} y}{V_{1}+V_{2}}, \\
y^{\prime}=\frac{y V_{1} V_{2}}{V_{1}+V_{2}}\left(\alpha_{1} \beta_{1}+\alpha_{2} \beta_{2}\right)-H .
\end{gathered}
$$

\subsection{The Switching Functions}

The functions $\frac{\beta_{1} V_{1} y}{1+\left(\frac{V_{1}}{V_{2}}\right)^{n}}$ and $\frac{\beta_{2} V_{2} y}{1+\left(\frac{V_{2}}{V_{1}}\right)^{n}}$ have the characteristic property of the switching mechanism. When one of the prey population becomes large, the predator switches the predation to the other species. Thus switching behaviour occurs where the predators attack the habitat in which there are fewer preys.

$$
k_{1}=\frac{m \beta_{1} V_{1} y}{1+\left(\frac{V_{1}}{V_{2}}\right)^{n}}, \quad k_{2}=\frac{m \beta_{2} V_{2} y}{1+\left(\frac{V_{2}}{V_{1}}\right)^{n}} .
$$

When $V_{1} \gg V_{2}$ :

$$
k_{1}=\frac{m \beta_{1} V_{1} y}{1+\left(\frac{V_{1}}{V_{2}}\right)^{n}} \rightarrow 0, \quad k_{2}=\frac{m \beta_{2} V_{2} y}{1+\left(\frac{V_{2}}{V_{1}}\right)^{n}} \rightarrow m \beta_{2} V_{2} y
$$

When $V_{2} \gg V_{1}$ :

$$
k_{1}=\frac{m \beta_{1} V_{1} y}{1+\left(\frac{V_{1}}{V_{2}}\right)^{n}} \rightarrow m \beta_{1} V_{1} y, \quad k_{2}=\frac{m \beta_{2} V_{2} y}{1+\left(\frac{V_{2}}{V_{1}}\right)^{n}} \rightarrow 0 .
$$

\section{Constant Effort Harvesting $h(y)=h$}

\subsection{Boundedness}

The Boundedness of a system implies that the system is biologically well behaved. Boundedness may be interpreted as a natural restriction to growth due to limited resources. Let

$$
\varphi=V_{1}+V_{2}+y, R=\max \left(r_{1}, r_{2}\right), r=\min \left(r_{1}, r_{2}\right), K=\max \left(K_{1}, K_{2}\right) .
$$

and let $0<\eta<h$ be a constant. Also assume that $m>\alpha_{i}$ for $i=1,2$.

Adding Equation (5), Equation (6) and Equation (7):

$$
\begin{aligned}
\varphi^{\prime}+\eta \varphi & =V_{1}\left(r_{1}-\epsilon_{1}+\epsilon_{1} p_{12}+\eta\right)-\frac{r_{1} V_{1}^{2}}{K_{1}}+V_{2}\left(r_{2}-\epsilon_{2}+\epsilon_{2} p_{21}\right) \\
& -\frac{r_{2} V_{2}^{2}}{K_{2}}-\frac{V_{1} V_{2} y}{V_{1}+V_{2}}\left[\beta_{1}\left(m-\alpha_{1}\right)+\beta_{2}\left(m-\alpha_{2}\right)\right]-(h-\eta) y \\
& <V_{1}\left(r_{1}-\epsilon_{1}+\epsilon_{1} p_{12}+\eta\right)+V_{2}\left(r_{2}-\epsilon_{2}+\epsilon_{2} p_{21}+\eta\right)-\frac{r_{1} V_{1}^{2}}{K_{1}}-\frac{r_{2} V_{2}^{2}}{K_{2}} .
\end{aligned}
$$

Completing the square of the right hand side of the inequality and letting

$$
\tau_{1}=r_{1}-\epsilon_{1}+\epsilon_{1} p_{12}+\eta
$$

and

$$
\tau_{2}=r_{2}-\epsilon_{2}+\epsilon_{1} p_{21}+\eta
$$

leads to:

$$
\varphi^{\prime}+\eta \varphi \leqslant M
$$

where

$$
M=\frac{K_{1}}{4 r_{1}} \tau_{1}^{2}+\frac{K_{2}}{4 r_{2}} \tau_{2}^{2}
$$

is a positive constant which is written in terms of the parameters. This results in a differential inequality that can be solved leading to:

$$
0<\varphi \leqslant \frac{M}{\eta}\left(1-e^{-\eta t}\right)+M_{0} e^{-\eta t},
$$


where $M_{0}$ is the value of $M$ at $t=0$. As $t \rightarrow \infty, 0<\varphi \leqslant \frac{M}{\eta}$. Hence all trajectories are bounded.

\subsection{Equilibrium Points}

These are obtained by setting Equation (5), Equation (6) and Equation (7) equal to zero, and solving the resultant algebraic equations. Three possible cases - origin, boundary and interior equilibria - are found. We have:

$$
\begin{gathered}
\left(r_{1}-\epsilon_{1}\right) V_{1}-\frac{r_{1} V_{1}^{2}}{K_{1}}+\epsilon_{2} p_{21} V_{2}-\frac{m \beta_{1} V_{1} V_{2} y}{V_{1}+V_{2}}=0, \\
\left(r_{2}-\epsilon_{2}\right) V_{2}-\frac{r_{2} V_{2}^{2}}{K_{2}}+\epsilon_{1} p_{12} V_{1}-\frac{m \beta_{2} V_{1} V_{2} y}{V_{1}+V_{2}}=0, \\
\left(\frac{\alpha_{1} \beta_{1} V_{1} V_{2}}{V_{1}+V_{2}}+\frac{\alpha_{2} \beta_{2} V_{1} V_{2}}{V_{1}+V_{2}}-h\right) y=0 .
\end{gathered}
$$

From Equation (10), we see that either

$$
y=0,
$$

or

$$
\frac{\alpha_{1} \beta_{1} V_{1} V_{2}}{V_{1}+V_{2}}+\frac{\alpha_{2} \beta_{2} V_{1} V_{2}}{V_{1}+V_{2}}-h=0
$$

We study these two cases below.

3.2.1 Case 1: The Origin $E_{0}$ and the Boundary Equilibrium $E_{1}$

Substituting $y=0$ in Equation (8) and Equation (9), we get

$$
\begin{aligned}
& r_{1} V_{1}\left(1-\frac{V_{1}}{K_{1}}\right)+\epsilon_{2} p_{21} V_{2}-\epsilon_{1} V_{1}=0 \\
& r_{2} V_{2}\left(1-\frac{V_{2}}{K_{2}}\right)+\epsilon_{1} p_{12} V_{1}-\epsilon_{2} V_{2}=0
\end{aligned}
$$

One solution is at the origin $E_{0}$ when

$$
V_{1}=V_{2}=y=0 .
$$

Using Equation (12), another equilibrium value $E_{1}$ may be found at

$$
V_{2}=V_{1}\left(\frac{r_{1}}{\epsilon_{2} p_{21} K_{1}} V_{1}-\frac{r_{1}-\epsilon_{1}}{\epsilon_{2} p_{21}}\right)
$$

where

$$
V_{1}>\frac{\left(r_{1}-\epsilon_{1}\right) K_{1}}{r_{1}} .
$$

Substituting for $V_{2}$ in Equation (13) leads to an equation of the form:

$$
b_{1} V_{1}^{3}+b_{2} V_{1}^{2}+b_{3} V_{1}++b_{4}=0
$$

where

$$
\begin{gathered}
b_{1}=\frac{r_{1}^{2} r_{2}}{\epsilon_{2}^{2} p_{21}^{2} K_{1}^{2} K_{2}}>0, \\
b_{2}=-\frac{2 r_{1} r_{2}\left(r_{1}-\epsilon_{1}\right)}{\epsilon_{2}^{2} p_{21}^{2} K_{1} K_{2}}<0, \\
b_{3}=\frac{\left(r_{1}-\epsilon_{1}\right)^{2} r_{2}}{\epsilon_{2}^{2} p_{21}^{2} K_{2}}-\frac{\left(r_{2}-\epsilon_{2}\right) r_{1}}{\epsilon_{2} p_{21} K_{1}}, \\
b_{4}=\frac{\left(r_{2}-\epsilon_{2}\right)\left(r_{1}-\epsilon_{1}\right)}{\epsilon_{2} p_{21}}-\epsilon_{1} p_{12} .
\end{gathered}
$$


Descartes' Rule of Signs indicates that a unique positive solution occurs when

$$
\frac{\left(r_{1}-\epsilon_{1}\right)^{2} r_{2}}{\epsilon_{2}^{2} p_{21}^{2} K_{2}}<\frac{\left(r_{2}-\epsilon_{2}\right) r_{1}}{\epsilon_{2} p_{21} K_{1}}
$$

and

$$
\frac{\left(r_{2}-\epsilon_{2}\right)\left(r_{1}-\epsilon_{1}\right)}{\epsilon_{2} p_{21}}<\epsilon_{1} p_{12}
$$

Using Equation (14), $V_{2}$ can be calculated.

3.2.2 Case 2: The Interior Coexistence Equilibrium $E_{2}$

Solving Equation (11) for $V_{2}$ :

$$
V_{2}=\frac{h V_{1}}{\left(\alpha_{1} \beta_{1}+\alpha_{2} \beta_{2}\right) V_{1}-h},
$$

where

$$
V_{1}>\frac{h}{\alpha_{1} \beta_{1}+\alpha_{2} \beta_{2}} .
$$

To simplify, let

$$
\phi h=\alpha_{1} \beta_{1}+\alpha_{2} \beta_{2}
$$

to get

$$
V_{2}=\frac{V_{1}}{\phi V_{1}-1} .
$$

Substituting in Equation(8) and using Equation(11), we get

$$
y=\left(\left(r_{1}-\epsilon_{1}\right)-\frac{r_{1} V_{1}}{K_{1}}+\frac{\epsilon_{2} p_{21}}{\phi V_{1}-1}\right) \frac{\phi V_{1}}{m \beta_{1}} .
$$

Substituting for $y$ and $V_{2}$ in Equation (9) leads to an equation of the form:

$$
a_{1} V_{1}^{3}+a_{2} V_{1}^{2}+a_{3} V_{1}+a_{4}=0,
$$

where

$$
\begin{gathered}
a_{1}=\frac{\beta_{2} r_{1} \phi^{2}}{K_{1} \beta_{1}}>0, \\
a_{2}=\phi^{2} \epsilon_{1} p_{12}-\frac{2 r_{1} \beta_{2}}{K_{1} \beta_{1}} \phi-\frac{\beta_{2}}{\beta_{1}} \phi^{2}\left(r_{1}-\epsilon_{1}\right), \\
a_{3}=\frac{\beta_{2} r_{1}}{\beta_{1} K_{1}}+\phi\left(r_{2}-\epsilon_{2}\right)-2 \phi \epsilon_{1} p_{12}+\frac{2 \phi \beta_{2}}{\beta_{1}}\left(r_{1}-\epsilon_{1}\right)-\frac{\phi}{\beta_{1}} \beta_{2} \epsilon_{2} p_{21}-\frac{r_{2}}{K_{2}}, \\
a_{4}=\epsilon_{1} p_{12}-\left(r_{2}-\epsilon_{2}\right)-\frac{\beta_{2}}{\beta_{1}}\left(r_{1}-\epsilon_{1}-\epsilon_{2} p_{21}\right) .
\end{gathered}
$$

This can be solved for $V_{1}$, and hence for $y$ and $V_{2}$.

\subsection{Stability Analysis}

We are interested in the asymptotic stability of the steady states of system. We linearise the system about its steady states and determine the associated characteristic equation. We use the following two conditions in order to determine stability of the different equilibria. For the eigenvalues of a $2 \times 2$ matrix to have negative real part, its determinant must be positive and its trace must be negative. For a $3 \times 3$ matrix, the Routh-Hurwitz conditions are used.

The Jacobian matrix $J$ is found to be

$$
\left[\begin{array}{ccc}
\left(r_{1}-\epsilon_{1}\right)-\frac{2 V_{1} r_{1}}{K_{1}}-\frac{m \beta_{1} y V_{2}^{2}}{\left(V_{1}+V_{2}\right)^{2}} & \epsilon_{2} p_{21}-\frac{m \beta_{1} y V_{1}^{2}}{\left(V_{1}+V_{2}\right)^{2}} & -\frac{m \beta_{1} V_{1} V_{2}}{V_{1}+V_{2}} \\
\epsilon_{1} p_{12}-\frac{m \beta_{2} y V_{2}^{2}}{\left(V_{1}+V_{2}\right)^{2}} & \left(r_{2}-\epsilon_{2}\right)-\frac{2 V_{2} r_{2}}{K_{2}}-\frac{m \beta_{2} y V_{1}^{2}}{\left(V_{1}+V_{2}\right)^{2}} & -\frac{m \beta_{2} V_{1} V_{2}}{V_{1}+V_{2}} \\
\frac{\alpha_{1} \beta_{1} y V_{2}^{2}}{\left(V_{1}+V_{2}\right)^{2}}+\frac{\alpha_{2} \beta_{2} y V_{2}^{2}}{\left(V_{1}+V_{2}\right)^{2}} & \frac{\alpha_{1} \beta_{1} y V_{1}^{2}}{\left(V_{1}+V_{2}\right)^{2}}+\frac{\alpha_{2} \beta_{2} y V_{1}^{2}}{\left(V_{1}+V_{2}\right)^{2}} & \frac{\alpha_{1} \beta_{1} V_{1} V_{2}}{V_{1}+V_{2}}+\frac{\alpha_{2} \beta_{2} V_{1} V_{2}}{V_{1}+V_{2}}-h
\end{array}\right]
$$


3.3.1 Case $E_{0}: V_{1}=V_{2}=y=0$

Here, Jacobian $J$ reduces to

$$
\left[\begin{array}{ccc}
r_{1}-\epsilon_{1} & \epsilon_{2} p_{21} & 0 \\
\epsilon_{1} p_{12} & r_{2}-\epsilon_{2} & 0 \\
0 & 0 & -h
\end{array}\right]
$$

with eigenvalues $-h$, and the eigenvalues of

$$
\left[\begin{array}{cc}
\left(r_{1}-\epsilon_{1}\right) & \epsilon_{2} p_{21} \\
\epsilon_{1} p_{12} & \left(r_{2}-\epsilon_{2}\right)
\end{array}\right]
$$

The characteristic polynomial of this is

$$
X^{2}-\tau X+\operatorname{det}=0
$$

with trace:

$$
\tau=\left(r_{1}-\epsilon_{1}\right)+\left(r_{2}-\epsilon_{2}\right)>0
$$

and determinant:

$$
\operatorname{det}=\left(r_{1}-\epsilon_{1}\right)\left(r_{2}-\epsilon_{2}\right)-\epsilon_{1} \epsilon_{2} p_{12} p_{21} .
$$

Hence there will be at least one positive eigenvalue. Thus this case is always unstable.

3.3.2 Case $E_{1}: y=0, V_{1} \neq 0, V_{2} \neq 0$

Here, the Jacobian $J$ reduces to

$$
\left[\begin{array}{ccc}
\left(r_{1}-\epsilon_{1}\right)-\frac{2 V_{1} r_{1}}{K_{1}} & \epsilon_{2} p_{21} & -\frac{m \beta_{1} V_{1} V_{2}}{V_{1}+V_{2}} \\
\epsilon_{1} p_{12} & \left(r_{2}-\epsilon_{2}\right)-\frac{2 V_{2} r_{2}}{K_{2}} & -\frac{m \beta_{2} V_{1} V_{2}}{V_{1}+V_{2}} \\
0 & 0 & V_{1} V_{2} \frac{\alpha_{1} \beta_{1}+\alpha_{2} \beta_{2}}{V_{1}+V_{2}}-h
\end{array}\right]
$$

The eigenvalues are found to be

$$
V_{1} V_{2} \frac{\alpha_{1} \beta_{1}+\alpha_{2} \beta_{2}}{V_{1}+V_{2}}-h
$$

and the eigenvalues of

$$
\left[\begin{array}{cc}
r_{1}-\frac{2 V_{1} r_{1}}{K_{1}}-\epsilon_{1} & \epsilon_{2} p_{21} \\
\epsilon_{1} p_{12} & r_{2}-\frac{2 V_{2} r_{2}}{K_{2}}-\epsilon_{2}
\end{array}\right]
$$

Using Equation (12) and Equation (13) and substituting in $J$, we get:

$$
\left[\begin{array}{cc}
-\epsilon_{2} p_{21} \frac{V_{2}}{V_{1}}-\frac{V_{1} r_{1}}{K_{1}} & \epsilon_{2} p_{21} \\
\epsilon_{1} p_{12} & -\epsilon_{1} p_{12} \frac{V_{1}}{V_{2}}-\frac{V_{2} r_{2}}{K_{2}}
\end{array}\right]
$$

The characteristic polynomial here is

$$
X^{2}-\tau X+\operatorname{det}=0
$$

with trace

$$
\tau=-\epsilon_{2} p_{21} \frac{V_{2}}{V_{1}}-\frac{V_{1} r_{1}}{K_{1}}-\epsilon_{1} p_{12} \frac{V_{1}}{V_{2}}-\frac{V_{2} r_{2}}{K_{2}}<0
$$

and determinant

$$
\operatorname{det}=\left(\epsilon_{2} p_{21} \frac{V_{2}}{V_{1}}+\frac{V_{1} r_{1}}{K_{1}}\right)\left(\epsilon_{1} p_{12} \frac{V_{1}}{V_{2}}+\frac{V_{2} r_{2}}{K_{2}}\right)-\epsilon_{1} \epsilon_{2} p_{12} p_{21}>0 .
$$

This means that there are two negative eigenvalues. However, the stability depends on whether

$$
V_{1} V_{2} \frac{\alpha_{1} \beta_{1}+\alpha_{2} \beta_{2}}{V_{1}+V_{2}}<h
$$


3.3.3 Case $E_{2}: V_{2}=\frac{V_{1}}{\phi V_{1}-1}, y=\left(\left(r_{1}-\epsilon_{1}\right)-\frac{r_{1} V_{1}}{K_{1}}+\frac{\epsilon_{2} p_{21}}{\phi V_{1}-1}\right) \frac{\phi V_{1}}{m \beta_{1}}$

When

$$
\frac{\alpha_{1} \beta_{1} V_{1} V_{2}}{V_{1}+V_{2}}+\frac{\alpha_{2} \beta_{2} V_{1} V_{2}}{V_{1}+V_{2}}-h(y)=0,
$$

the Jacobian $J$ reduces to:

$$
\left[\begin{array}{ccc}
\left(r_{1}-\epsilon_{1}\right)-\frac{2 V_{1} r_{1}}{K_{1}}-\frac{m \beta_{1} y V_{2}^{2}}{\left(V_{1}+V_{2}\right)^{2}} & \epsilon_{2} p_{21}-\frac{m \beta_{1} y V_{1}^{2}}{\left(V_{1}+V_{2}\right)^{2}} & -\frac{m \beta_{1}}{\phi} \\
\epsilon_{1} p_{12}-\frac{m \beta_{2} y V_{2}^{2}}{\left(V_{1}+V_{2}\right)^{2}} & \left(r_{2}-\epsilon_{2}\right)-\frac{2 V_{2} r_{2}}{K_{2}}-\frac{m \beta_{2} y V_{1}^{2}}{\left(V_{1}+V_{2}\right)^{2}} & -\frac{m \beta_{2}}{\phi} \\
\frac{h y}{\phi V_{1}^{2}} & \frac{h y}{\phi V_{2}^{2}} & 0
\end{array}\right] .
$$

From Equation (8), we can show that

$$
\epsilon_{2} p_{21}-\frac{m \beta_{1} y V_{1}^{2}}{\left(V_{1}+V_{2}\right)^{2}}=-\frac{V_{1}}{V_{2}}\left(q-\frac{r_{1} V_{1}}{K_{1}}\right),
$$

where

$$
q=\left(r_{1}-\epsilon_{1}\right)-\frac{m y \beta_{1} V_{2}^{2}}{\left(V_{1}+V_{2}\right)^{2}} .
$$

Similarly using Equation (9), we have

$$
\epsilon_{1} p_{12}-\frac{m \beta_{2} y V_{2}^{2}}{\left(V_{1}+V_{2}\right)^{2}}=-\frac{V_{2}}{V_{1}}\left(s-\frac{r_{2} V_{2}}{K_{2}}\right),
$$

where

$$
s=\left(r_{2}-\epsilon_{2}\right)-\frac{m y \beta_{2} V_{1}^{2}}{\left(V_{1}+V_{2}\right)^{2}} .
$$

Substituting these expressions in $J$, and letting

$$
\begin{gathered}
L=\left(\phi V_{1}-1\right)>0, \\
M=q-\frac{2 V_{1} r_{1}}{K_{1}}=\left(r_{1}-\epsilon_{1}\right)-\frac{2 V_{1} r_{1}}{K_{1}}-\frac{m \beta_{1} y}{\phi^{2} V_{1}^{2}}, \\
N=s-\frac{2 V_{2} r_{2}}{K_{2}}=\left(r_{2}-\epsilon_{2}\right)-\frac{2 V_{2} r_{2}}{K_{2}}-\frac{m \beta_{2} y\left(\phi V_{1}-1\right)^{2}}{\phi^{2} V_{1}^{2}}, \\
Q=\frac{h y}{\phi V_{1}^{2}}>0,
\end{gathered}
$$

we get

$$
J=\left[\begin{array}{ccc}
M & -L\left(M+\frac{r_{1} V_{1}}{K_{1}}\right) & -\frac{m \beta_{1}}{\phi} \\
-\frac{1}{L}\left(N+\frac{r_{2} V_{1}}{L K_{2}}\right) & N & -\frac{m \beta_{2}}{\phi} \\
Q & Q L^{2} & 0
\end{array}\right] .
$$

The corresponding characteristic polynomial has the form:

$$
X^{3}+b_{1} X^{2}+b_{2} X+b_{3}=0
$$

where

$$
\begin{gathered}
b_{1}=(-M-N)=\frac{2}{K_{1}} V_{1} r_{1}-s-q+\frac{2}{K_{2}} V_{2} r_{2}, \\
b_{2}=M N-\left(N+\frac{1}{L K_{2}} V_{1} r_{2}\right)\left(M+\frac{1}{K_{1}} V_{1} r_{1}\right)+Q \frac{m}{\phi} \beta_{1}+L^{2} Q \frac{m}{\phi} \beta_{2},
\end{gathered}
$$




$$
b_{3}=\left(\begin{array}{c}
Q\left(M \frac{m}{\phi} \beta_{1}-L \frac{m}{\phi} \beta_{2}\left(M+\frac{1}{K_{1}} V_{1} r_{1}\right)\right)-\left(Q \frac{m}{\phi} \beta_{1}+L^{2} Q \frac{m}{\phi} \beta_{2}\right)(M+N) \\
+L^{2} Q\left(N \frac{m}{\phi} \beta_{2}-\frac{1}{L} \frac{m}{\phi} \beta_{1}\left(N+\frac{1}{L K_{2}} V_{1} r_{2}\right)\right)
\end{array}\right) .
$$

Using the Routh-Hurwitz criteria, the eigenvalues will have negative real parts if

$$
b_{1}>0, b_{3}>0, b_{1} b_{2}-b_{3}>0
$$

for stability. Now if $b_{1}>0$, then we have

$$
\frac{2}{K_{1}} V_{1} r_{1}+\frac{2}{K_{2}} V_{2} r_{2}>s+q
$$

Since we know that

$$
s+q=-\frac{y m}{\phi^{2}}\left(\frac{\beta_{1}}{V_{2}^{2}}+\frac{\beta_{2}}{V_{1}^{2}}\right)+\left(r_{1}-\epsilon_{1}\right)+\left(r_{2}-\epsilon_{2}\right),
$$

it follows that if $b_{1}>0$, we get

$$
r_{1}\left(\frac{2}{K_{1}} V_{1}-1\right)+r_{2}\left(\frac{2}{K_{2}} V_{2}-1\right)+m y \frac{\beta_{1} V_{2}^{2}+\beta_{2} V_{1}^{2}}{V_{1}^{2} V_{2}^{2}}+\epsilon_{1}+\epsilon_{2}>0,
$$

where

$$
\begin{gathered}
b_{2}=\frac{1}{L \phi K_{1} K_{2}}\left(Q m \beta_{2} K_{1} K_{2} L^{3}-N \phi K_{2} r_{1} L V_{1}+Q m \beta_{1} K_{1} K_{2} L-\phi r_{1} r_{2} V_{1}^{2}-M \phi K_{1} r_{2} V_{1}\right), \\
b_{3}=-\frac{Q m}{\phi K_{1} K_{2}}\left(\beta_{1} K_{1} V_{1} r_{2}+L \beta_{2} K_{2} V_{1} r_{1}+K_{1} K_{2}(L+1)\left(N \beta_{1}+L M \beta_{2}\right)\right) .
\end{gathered}
$$

Given that $\frac{Q m}{\phi K_{1} K_{2}}>0$, when $b_{3}>0$ we have

$$
\beta_{1} K_{1} V_{1} r_{2}+L \beta_{2} K_{2} V_{1} r_{1}+K_{1} K_{2}(L+1)\left(N \beta_{1}+L M \beta_{2}\right)<0
$$

and

$$
b_{1} b_{2}-b_{3}=\frac{1}{\phi K_{1} K_{2} L}\left(\begin{array}{c}
L Q m K_{1} K_{2}\left(\beta_{1}-L \beta_{2}\right)(-M+L N) \\
+\phi V_{1}(M+N)\left(M K_{1} r_{2}+V_{1} r_{1} r_{2}+L N K_{2} r_{1}\right) \\
+L Q m V_{1}\left(\beta_{1} K_{1} r_{2}+L \beta_{2} K_{2} r_{1}\right)
\end{array}\right) .
$$

For $b_{1} b_{2}-b_{3}>0$ since $\frac{1}{\phi K_{1} K_{2} L}>0$, it follows that

$$
\left(\begin{array}{c}
L Q m K_{1} K_{2}\left(\beta_{1}-L \beta_{2}\right)(L N-M) \\
+\phi V_{1}(M+N)\left(M K_{1} r_{2}+V_{1} r_{1} r_{2}+L N K_{2} r_{1}\right) \\
+L Q m V_{1}\left(\beta_{1} K_{1} r_{2}+L \beta_{2} K_{2} r_{1}\right)
\end{array}\right)>0 .
$$

\subsection{Numerical Analysis}

We consider numerical simulations using the three datasets in Table 2, chosen so as to reflect different scenarios associated with crime. The model is run for three datasets - a base Dataset 1- and two other Datasets (Dataset 2 and Dataset 3), one representing a decrease in the conversion rate of the prey, and the other representing $r_{j}<\epsilon_{j}$ for $j=1,2$. For the base dataset, there was a decrease in both prey populations with the criminals or predators eventually outnumbering both prey populations. Decreasing the rate of incoming predators as in Dataset 2, leads to an increase in prey populations and a smaller predator population. If the emigration rate is higher than the growth rate of the prey - Dataset 3, this leads to few people remaining in the prey habitats or villages. These equilibrium values are as given in Table 3. Figures 1-3 show the time series of the populations for the different datasets, which all tend to a stable equilibrium value as shown by the eigenvalues. 
Table 2. Datasets 1-3

\begin{tabular}{cccc}
\hline Parameter & Dataset 1 & Dataset 2 & Dataset 3 \\
\hline$r_{1}$ & 0.75 & 0.75 & 0.2 \\
$r_{2}$ & 0.7 & 0.7 & 0.4 \\
$K_{1}$ & 100 & 100 & 100 \\
$K_{2}$ & 150 & 150 & 150 \\
$\epsilon_{1}$ & 0.7 & 0.7 & 0.7 \\
$\epsilon_{2}$ & 0.65 & 0.65 & 0.65 \\
$p_{12}$ & 0.9 & 0.9 & 0.9 \\
$p_{21}$ & 0.9 & 0.9 & 0.9 \\
$m$ & 0.5 & 0.5 & 0.5 \\
$\alpha_{1}$ & 0.5 & 0.009 & 0.5 \\
$\alpha_{2}$ & 0.4 & 0.006 & 0.4 \\
$\beta_{1}$ & 0.3 & 0.3 & 0.3 \\
$\beta_{2}$ & 0.5 & 0.5 & 0.5 \\
$h$ & 0.2 & 0.2 & 0.2 \\
\hline
\end{tabular}

Table 3. Equilibrium values for Datasets 1-3

\begin{tabular}{cccc}
\hline Equilibrium Values & Dataset 1 & Dataset 2 & Dataset 3 \\
\hline$V_{1}$ (Prey) & 1.5111 & 67.1733 & 1.0499 \\
$V_{2}$ (Prey) & 0.9194 & 73.4575 & 1.2539 \\
$y$ (Predator) & 6.9562 & 2.3726 & 2.4076 \\
eigenvalue 1 & -0.6639 & -1.4198 & -0.9923 \\
eigenvalue 2 & $-0.0444+0.3333 i$, & $-0.2029+0.0794 i$ & $-0.0003+0.2209 i$ \\
eigenvalue 3 & $-0.0444-0.3333 i$ & $-0.2029-0.0794 i$ & $-0.0003-0.2209 i$ \\
stability & stable & stable & stable \\
\hline
\end{tabular}
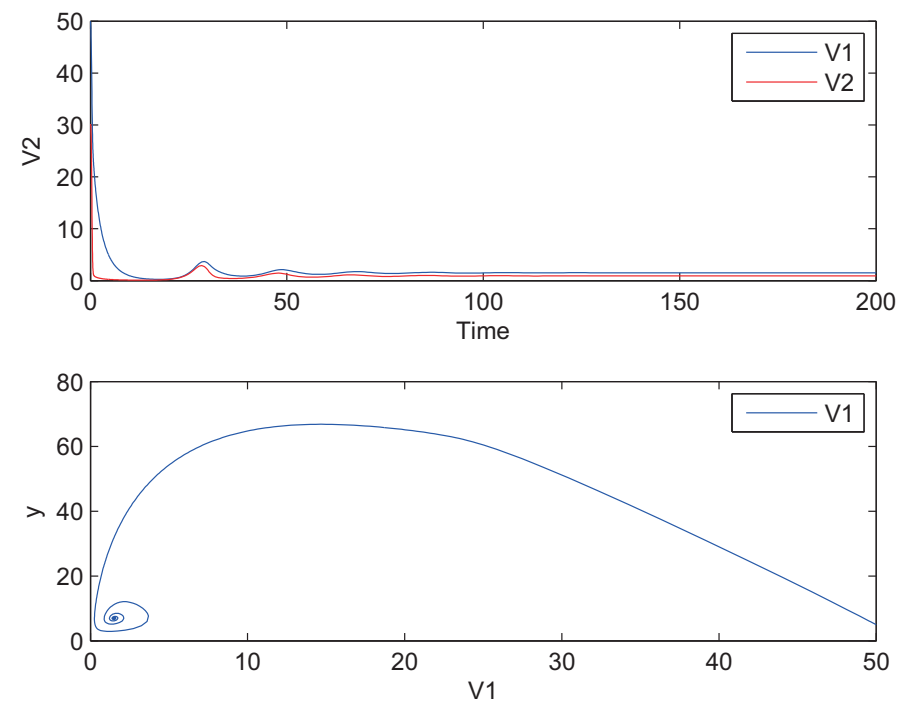

Figure 1. Timeseries for Dataset 1 


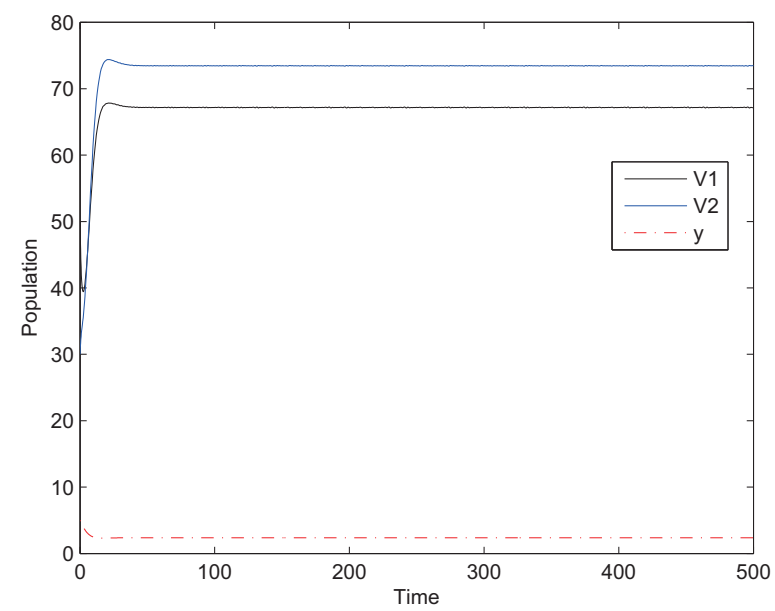

Figure 2. Timeseries for Dataset 2

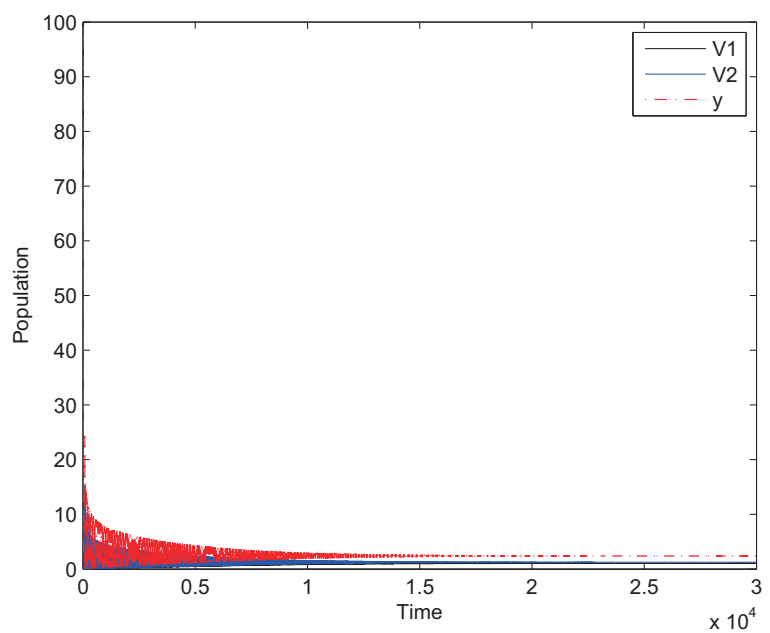

Figure 3. Timeseries for Dataset 3

\subsection{Bifurcation Analysis}

The main objective in this section is to show numerically that all the three species can coexist either in the form of oscillatory solution (stable limit cycle) or in the form of a steady state solution (stable focus or node) for some range of parameters.

\subsubsection{Dataset 1}

Hopf bifurcations were found on varying $r_{1}, r_{2}, \epsilon_{1}$ and $\epsilon_{2}$. The bifurcation points are given in Table 4 .

Table 4. Hopf Bifurcation Points for Dataset 1

\begin{tabular}{cll}
\hline Parameter & \multicolumn{2}{l}{ Hopf Bifurcation } \\
\hline$r_{1}$ & 0.276759 & Hopf -1st lyapunov coefficient $>0$ (subcritical) \\
& 0.511503 & Hopf - 1st lyapunov coefficient $<0$ (supercritical) \\
\hline$r_{2}$ & 1.031463 & Hopf - 1st lyapunov coefficient $<0$ (supercritical) \\
& 1.287846 & Hopf - 1st lyapunov coefficient $<0$ (supercritical) \\
\hline$\varepsilon_{1}$ & 0.908070 & Hopf -1st lyapunov coefficient $>0$ (subcritical) \\
& 0.982621 & Hopf - 1st lyapunov coefficient $<0$ (supercritical) \\
\hline$\epsilon_{2}$ & 0.419703 & Hopf -1st lyapunov coefficient $>0$ (subcritical) \\
& 0.484760 & Hopf - 1st lyapunov coefficient $<0$ (supercritical) \\
\hline
\end{tabular}




\subsubsection{Dataset 2}

Transcritical bifurcation are found as shown in Table 5. At the transcritical points, the equilibrium shifts from the coexistence equilibrium to the boundary one. There are no Hopf bifurcations.

Table 5. Bifurcation Points for Dataset 2

\begin{tabular}{ccc}
\hline Parameter & BP & Region of coexistence \\
\hline$\epsilon_{1}$ & 0.082042 & $>\mathrm{BP}$ \\
$\epsilon_{2}$ & 0.082991 & $>\mathrm{BP}$ \\
$\beta_{1}$ & 0.068311 & $>\mathrm{BP}$ \\
$\beta_{2}$ & 0.152466 & $>\mathrm{BP}$ \\
$h$ & 0.315371 & $<\mathrm{BP}$ \\
$p_{12}$ & 0.322527 & $>\mathrm{BP}$ \\
$p_{21}$ & 0.349059 & $>\mathrm{BP}$ \\
\hline
\end{tabular}

\subsubsection{Dataset 3}

Hopf bifurcations are found on varying $\alpha_{1}, \alpha_{2}, \beta_{1}$ and $\beta_{2}$ as shown in Table 6.

Table 6. Bifurcation points for Dataset 3

\begin{tabular}{cll}
\hline Parameter & \multicolumn{2}{l}{ Hopf Bifurcation } \\
\hline$\alpha_{1}$ & 0.276759 & Hopf -1st lyapunov coefficient $>0$ (subcritical) \\
$\alpha_{2}$ & 0.530483 & Hopf - 1st lyapunov coefficient $<0$ (supercritical) \\
$\beta_{1}$ & 0.140039 & Hopf -1st lyapunov coefficient $>0$ (subcritical) \\
& 0.272108 & Hopf - 1st lyapunov coefficient $<0$ (supercritical) \\
$\beta_{2}$ & 0.529815 & Hopf -1st lyapunov coefficient $>0$ (subcritical) \\
\hline
\end{tabular}

\section{Constant Rate or Constant Yield Harvesting: $h(y)=\frac{H}{y}$}

The system is now analysed for $n=1$ and $h(y)=\frac{H}{y}$. The system becomes:

$$
\begin{gathered}
V_{1}^{\prime}=\left(r_{1}-\epsilon_{1}\right) V_{1}-\frac{r_{1} V_{1}^{2}}{K_{1}}+\epsilon_{2} p_{21} V_{2}-\frac{m \beta_{1} V_{1} V_{2} y}{V_{1}+V_{2}}, \\
V_{2}^{\prime}=\left(r_{2}-\epsilon_{2}\right) V_{2}-\frac{r_{2} V_{2}^{2}}{K_{2}}+\epsilon_{1} p_{12} V_{1}-\frac{m \beta_{2} V_{1} V_{2} y}{V_{1}+V_{2}}, \\
y^{\prime}=\frac{y V_{1} V_{2}}{V_{1}+V_{2}}\left(\alpha_{1} \beta_{1}+\alpha_{2} \beta_{2}\right)-H .
\end{gathered}
$$

\subsection{Equilibrium Points and Stability}

These are obtained by setting the system equations equal to zero, and solving the resultant algebraic equations. Unlike the previous case, there is only one equilibrium and no equilibrium at the origin or boundary equilibrium. We consider

$$
\begin{gathered}
\left(r_{1}-\epsilon_{1}\right) V_{1}-\frac{r_{1} V_{1}^{2}}{K_{1}}+\epsilon_{2} p_{21} V_{2}-\frac{m \beta_{1} V_{1} V_{2} y}{V_{1}+V_{2}}=0, \\
\left(r_{2}-\epsilon_{2}\right) V_{2}-\frac{r_{2} V_{2}^{2}}{K_{2}}+\epsilon_{1} p_{12} V_{1}-\frac{m \beta_{2} V_{1} V_{2} y}{V_{1}+V_{2}}=0, \\
\left(\frac{\alpha_{1} \beta_{1} V_{1} V_{2}}{V_{1}+V_{2}}+\frac{\alpha_{2} \beta_{2} V_{1} V_{2}}{V_{1}+V_{2}}\right) y=H .
\end{gathered}
$$

From Equation (21),

$$
y=\frac{H\left(V_{1}+V_{2}\right)}{V_{1} V_{2}\left(\alpha_{1} \beta_{1}+\alpha_{2} \beta_{2}\right)}=\frac{\left(V_{1}+V_{2}\right)}{\phi V_{1} V_{2}}>0,
$$


where

$$
\frac{1}{\phi}=\frac{H}{\alpha_{1} \beta_{1}+\alpha_{2} \beta_{2}} .
$$

Substituting for $y$ in Equation (19) we can get an expression for $V_{2}$ :

$$
V_{2}=\frac{1}{\epsilon_{2} p_{21}}\left(\frac{r_{1} V_{1}^{2}}{K_{1}}-\left(r_{1}-\epsilon_{1}\right) V_{1}+k_{1}\right)
$$

where $\frac{m \beta_{1}}{\phi}=k_{1}$. When $V_{2}>0$,

$$
V_{1}^{2}-\frac{\left(r_{1}-\epsilon_{1}\right) K_{1}}{r_{1}} V_{1}+k_{1} \frac{K_{1}}{r_{1}}>0
$$

which means that either

$$
\frac{\left(r_{1}-\epsilon_{1}\right) K_{1}}{r_{1}}+\left(\frac{\left(r_{1}-\epsilon_{1}\right)^{2} K_{1}^{2}}{r_{1}^{2}}-4 k_{1} \frac{K_{1}}{r_{1}}\right)^{\frac{1}{2}}>0
$$

or

$$
\frac{\left(r_{1}-\epsilon_{1}\right) K_{1}}{r_{1}}-\left(\frac{\left(r_{1}-\epsilon_{1}\right)^{2} K_{1}^{2}}{r_{1}^{2}}-4 k_{1} \frac{K_{1}}{r_{1}}\right)^{\frac{1}{2}}<0 .
$$

Using Equations (20) and (23), we obtain an equation of the form

$$
c_{1} V_{1}^{4}+c_{2} V_{1}^{3}+c_{3} V_{1}^{2}+c_{4} V_{1}+c_{5}=0
$$

where:

$$
\begin{gathered}
c_{1}=\frac{1}{\epsilon_{2}^{2} K_{1}^{2} K_{2}} \frac{1}{p_{21}^{2}}\left(r_{1}^{2} r_{2}\right)>0, \\
c_{2}=\frac{2}{\epsilon_{2}^{2} K_{1} K_{2}} r_{1} \frac{r_{2}}{p_{21}^{2}}\left(\epsilon_{1}-r_{1}\right), \\
c_{3}=\frac{1}{\epsilon_{2}^{2} K_{2}} \frac{r_{2}}{p_{21}^{2}}\left(\left(\epsilon_{1}-r_{1}\right)^{2}+\frac{2}{K_{1}} k_{1} r_{1}\right)+\frac{1}{\epsilon_{2} K_{1}} \frac{r_{1}}{p_{21}}\left(\epsilon_{2}-r_{2}\right), \\
c_{4}=-\epsilon_{1} p_{12}+\frac{1}{\epsilon_{2} p_{21}}\left(\epsilon_{1}-r_{1}\right)\left(\epsilon_{2}-r_{2}\right)+\frac{2}{\epsilon_{2}^{2} K_{2}} k_{1} \frac{r_{2}}{p_{21}^{2}}\left(\epsilon_{1}-r_{1}\right), \\
c_{5}=k_{2}+\frac{1}{\epsilon_{2}} \frac{k_{1}}{p_{21}}\left(\epsilon_{2}-r_{2}\right)+\frac{1}{\epsilon_{2}^{2} K_{2}} k_{1}^{2} \frac{r_{2}}{p_{21}^{2}} .
\end{gathered}
$$

Since this is a quartic equation there may be four, two or zero real solutions. The Jacobian matrix $J$ is

$$
\left[\begin{array}{ccc}
\left(r_{1}-\epsilon_{1}\right)-\frac{2 V_{1} r_{1}}{K_{1}-\frac{m \beta_{1} y V_{2}^{2}}{\left(V_{1}+V_{2}\right)^{2}}} & \epsilon_{2} p_{21}-\frac{m \beta_{1} y V_{1}^{2}}{\left(V_{1}+V_{2}\right)^{2}} & -\frac{m \beta_{1} V_{1} V_{2}}{V_{1}+V_{2}} \\
\epsilon_{1} p_{12}-\frac{m \beta_{2} y V_{2}^{2}}{\left(V_{1}+V_{2}\right)^{2}} & \left(r_{2}-\epsilon_{2}\right)-\frac{2 V_{2} r_{2}}{K_{2}}-\frac{m \beta_{2} y V_{1}^{2}}{\left(V_{1}+V_{2}\right)^{2}} & -\frac{m \beta_{2} V_{1} V_{2}}{V_{1}+V_{2}} \\
y V_{2}^{2} \frac{\alpha_{1} \beta_{1}+\alpha_{2} \beta_{2}}{\left(V_{1}+V_{2}\right)^{2}} & y V_{1}^{2} \frac{\alpha_{1} \beta_{1}+\alpha_{2} \beta_{2}}{\left(V_{1}+V_{2}\right)^{2}} & \frac{H}{y}
\end{array}\right] .
$$

This may be further simplified using Equation (22) and by letting

$$
s=\left(r_{2}-\epsilon_{2}\right)-\frac{m \beta_{2} y V_{1}^{2}}{\left(V_{1}+V_{2}\right)^{2}}, \quad q=\left(r_{1}-\epsilon_{1}\right)-\frac{m \beta_{1} y V_{2}^{2}}{\left(V_{1}+V_{2}\right)^{2}},
$$

to obtain

$$
J=\left[\begin{array}{ccc}
q-\frac{2 V_{1} r_{1}}{K_{1}} & -\frac{V_{1}}{V_{2}}\left(q-\frac{r_{1} V_{1}}{K_{1}}\right) & -\frac{m \beta_{1}}{\phi y} \\
-\frac{V_{2}}{V_{1}}\left(s-\frac{r_{2} V_{2}}{K_{2}}\right) & s-\frac{2 V_{2} r_{2}}{K_{2}} & -\frac{m \beta_{2}}{\phi y} \\
y V_{2}^{2} \frac{\phi H}{\left(V_{1}+V_{2}\right)^{2}} & y V_{1}^{2} \frac{\phi H}{\left(V_{1}+V_{2}\right)^{2}} & \frac{H}{y}
\end{array}\right] .
$$


The characteristic polynomial has the form:

$$
X^{3}+b_{1} X^{2}+b_{2} X+b_{3}=0
$$

where

$$
\begin{gathered}
b_{1}=\frac{2}{K_{1}} V_{1} r_{1}-s-\frac{H}{y}-q+\frac{2}{K_{2}} V_{2} r_{2} \\
b_{2}=-\frac{1}{y K_{1} K_{2}}\left(\begin{array}{c}
2 H K_{2} V_{1} r_{1}-H s K_{1} K_{2}-H q K_{1} K_{2}+2 H K_{1} V_{2} r_{2}-3 y V_{1} V_{2} r_{1} r_{2} \\
+q y K_{1} V_{2} r_{2}+s y K_{2} V_{1} r_{1}
\end{array}\right)+H m \frac{\beta_{1} V_{2}^{2}+\beta_{2} V_{1}^{2}}{\left(V_{1}+V_{2}\right)^{2}}, \\
b_{3}=\frac{1}{y K_{1} K_{2}}\left(H V_{2} r_{2}\left(q K_{1}-V_{1} r_{1}\right)+H V_{1} r_{1}\left(s K_{2}-V_{2} r_{2}\right)-H V_{1} V_{2} r_{1} r_{2}\right) \\
+\frac{H m}{K_{1} K_{2}\left(V_{1}+V_{2}\right)^{2}}\left(\begin{array}{c}
\beta_{1} K_{1} V_{2}^{3} r_{2}+\beta_{2} K_{2} V_{1}^{3} r_{1}+\beta_{1} K_{1} V_{2}\left(V_{1}+V_{2}\right)\left(V_{2} r_{2}-s K_{2}\right) \\
+\beta_{2} K_{2} V_{1}\left(V_{1}+V_{2}\right)\left(V_{1} r_{1}-q K_{1}\right)
\end{array}\right) .
\end{gathered}
$$

Using the Routh-Hurwitz criteria, the eigenvalues will have negative real parts (thus implying stability) when

$$
b_{1}>0, b_{3}>0, b_{1} b_{2}-b_{3}>0 .
$$

\subsection{Numerical Analysis}

Using the values in the previous models and keeping a constant harvest rate 0.2 , the only stable equilibrium values exist for Dataset 2 (see Table 7 and Figure 4). This results in a greater number of prey in $V_{2}$ than in $V_{1}$.

Table 7. Equilibrium values for constant yield harvesting

\begin{tabular}{ccccc}
\hline & Dataset 1 & Dataset 1 & Dataset 2 & Dataset 3 \\
\hline$V_{1}$ & 0.22 & 100.91 & 13.9005 & 0.43 \\
$V_{2}$ & 0.128 & 122.07 & 10.2935 & 0.5147 \\
$y$ & 7.05 & 0.010 & 5.9366 & 2.4392 \\
eigenvalue 1 & -0.7386 & -1.9118 & -0.7653 & -0.9876 \\
eigenvalue 2 & $0.0080+0.1183 i$ & -0.6412 & $-0.0283+0.1229 i$ & $0.0401+0.1359 i$ \\
eigenvalue 3 & $0.0080-0.1183 i$ & 19.3341 & $-0.0283-0.1229 i$ & $0.0401-0.1359 i$ \\
stability & unstable & unstable & stable & unstable \\
\hline
\end{tabular}

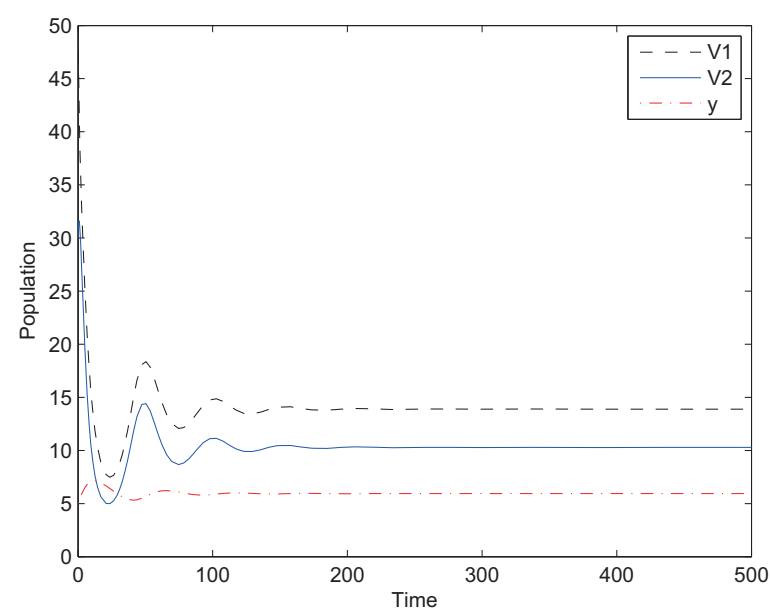

Figure 4. Timeseries for Dataset 2; Constant yield harvesting 


\subsection{Bifurcation Diagrams}

Using these values, the bifurcation points are found - Table 8. Hopf bifurcations in $\alpha_{1}$ and $\alpha_{2}$ occur for small values of these parameters - otherwise there are no stable equilibrium points. For $p / q / h$, the bifurcation points allow for the determination of Limit Points, beyond which there exists no equilibrium points.

Table 8. Bifurcation points for Dataset 2

\begin{tabular}{ccl}
\hline Parameter & Bifurcation Point & Region of coexistence \\
\hline$\alpha_{1}$ & 0.083418 & Hopf -1st lyapunov coefficient $>0$ (subcritical) \\
$\alpha_{2}$ & 0.050651 & Hopf - 1 st lyapunov coefficient $>0$ (subcritical) \\
$p$ & 0.372478 & $>$ Branch Point \\
$q$ & 0.301398 & $>$ Branch Point \\
$H$ & 0.5014 & $<$ Branch Point \\
\hline
\end{tabular}

\section{Discussion}

Like their animal counterparts, criminal predators often prey on the weak and vulnerable (Cooper et al., 2007). In the animal kingdom, one of the factors influencing group behaviour is the threat from predation (Heg et al., 2004)-there is safety in numbers. Group defense occurs when the prey work together to defend themselves against predators. In this model, we assume that criminals acting as predators seek out areas with smaller populations to prey upon. Thus there is a reduced hunting rate on the larger population. This may result in a strain on available resources in that population and may result in migration to the other area. Also, the other population may rush to its defense. This will then cause numbers there to increase and the predator switches predation to the smaller population. Thus switching behaviour occurs and the predator attacks the habitat in which there are fewer prey.

Our model differs in two ways from standard predator - prey models. Firstly the functional response includes a term $0 \leqslant m \leqslant 1$ that describes whether people or prey will leave the system after being attacked by criminals. $m=1$ corresponds to the situation in nature where prey are killed. In predator prey models, the numerical response describing the growth rate of the predator is usually proportional to the number of prey attacked. We use a similar numerical response since an increase in criminal activity in an area may lead to the area being perceived as an easy target and may result in a corresponding influx of criminals. Though this may result in an increase in security, when the temptation is great, there may be perpetrator displacement (Barr \& Pease, 1990) and an influx of new criminals. The model also includes a harvesting function $h(y)$, where the criminals are removed by the police.

The terms $\epsilon_{1} p_{12}$ and $\epsilon_{2} p_{21}$ represent the migration rate out of the area. $\epsilon_{1} p_{12}$ and $\epsilon_{2} p_{21}$ represent the probability of migrating to the other area. It is not unusual for people to leave an area when they feel threatened and migrate to a perceived safer area. In predator prey models, $\epsilon_{1} p_{12}$ and $\epsilon_{2} p_{21}$ indicates migration rates/inversion barrier strength in going from one habitat to the other where the probability of survival during a change of habitat may be less than one.

In constant effort harvesting, there are three equilibrium values - the origin $E_{0}$, the boundary equilibrium $E_{1}$ in which there are no criminals and the coexistence equilibrium $E_{2}$. Of these, $E_{0}$ is always unstable and there are conditions on the stability of the other two equilibria. This means that the prey population will never be driven to extinction - though the predators may be reduced to zero.

For Dataset 1, Hopf bifurcations are found on varying $r_{1}, r_{2}, \epsilon_{1}$ and $\epsilon_{2}$. This corresponds to the growth and the migration rates of the population. The Hopf bifurcation means for that range, the system exhibits unstable behavior and undergoes a transition from stationary to oscillatory behavior, and the prey populations will oscillate alternately. The system will become stable when the prey in one habitat outnumbers that in the other habitat. For Dataset 2, since the predator growth rate is small, both prey populations grow. The bifurcation points give the values at which a switch to the boundary equilibrium occurs. For a harvesting proportion, $h<0.315371$, it is possible to eliminate all the predators from the system. In Dataset 3 , where the prey migration rate is greater than the growth rate, hopf bifurcations are found on varying $\alpha_{1}, \alpha_{2}, \beta_{1}$ and $\beta_{2}$. These are related to the interaction between prey and predator. Both large $\alpha_{2}$ and large $\beta_{2}$ are unstable for a wide range of values $\alpha_{2}, \beta_{2}>0.53$. So an increase in the hunting or conversion rates may lead to oscillations in the predator and prey populations.

This second section analysed the same model as before, but used a constant harvesting function $H$. This form is used to describe the removal of prey when there is a quota (Brauer \& Castillo-Chavez, 2001). The numerical 
simulations showed that only Dataset 2 possessed stable equilibrium values. From Figure 4, we note that the populations exhibit oscillatory behavior for a certain time, and then they settle down at their equilibrium level.

Unlike constant effort harvesting, with constant rate or constant yield harvesting, it is not possible to reduce the number of predators to extinction since the only equilibrium is only the coexistence equilibrium. In both harvesting types, it is not possible for all the populations to go to extinction - the extinction equilibrium is also unstable in constant effort harvesting. Considering Dataset 2, a bifurcation analysis showed bifurcations for $\alpha_{1} / \alpha_{2} / p / q / h$. Comparing this with the constant effort harvesting function, a Hopf bifurcation occurs for $\alpha_{1}$ and $\alpha_{2}$. Assuming that the only parameter over which any control can be exerted is the harvesting one, then values beyond $H=0.5014$ will lead to unstable equilibrium values for constant rate harvesting and to the no-criminal equilibrium for the constant effort harvesting function $h=0.315371$. In the case of criminals attacking a population, the case of no-criminals is desirable. Hence changing the parameters to these values may be used to control the predators if a constant effort harvesting function is used.

\section{Conclusion}

Crime displacement, perpetrator displacement and group defense are important considerations in the crime fighting initiative. Displacement, rather than prevention, of crime is an unfortunate, unintended consequence of crime control (Barr \& Pease, 1990). We use an approach borrowed from mathematical ecology - predator prey systems with group defense, switching and harvesting. A harvest rate proportional to the population size (constant effort) or a constant harvest rate (constant yield) is studied to determine the best harvesting strategies of the predators. The Constant effort strategy may result in an additional equilibrium in which the predators or criminals are extinct. This equilibrium is not present in the Constant yield harvesting strategy where the only equilibrium is the coexistence one. Also, depending on parameter values a stable equilibrium or a limit cycle orbit is possible with both harvesting types.

\section{References}

Barr, R., \& Pease, K. (1990). Crime and justice: A review of research. Crime Placement, Displacement and Deflection, 12. University of Chicago Press.

Bhatt, B., Khan, Q., \& Jaju, R. (2005). Switching effect of predation on prey species living in two habitats exhibiting group defense. Proceedings of the 2005 WSEAS International Conference on Dynamical Systems and Control, CONTROL'05, Venice, Italy.

Bhattacharyya, R., \& Mukhopadhyay, B. (2006). Spatial dynamics of nonlinear prey migration and predator switching. Ecological Complexity, 3(2), 160-169. http://dx.doi.org/10.1016/j.ecocom.2006.01.001

Brauer, F., \& Castillo-Chavez, C. (2001). Mathematical Models in Population Biology and Epidemiology. Texts in Applied Mathematics, Springer.

Cooper, G. M., King, M. R., \& McHoes, T. (2007). Predators: Who are they and how to stop them. Prometheus Books.

Eck, J. (1993). The threat of crime displacement. Criminal Justice Abstracts, 25, 527-546.

Heg, D., Bachar, Z., Brouwer, L., \& Taborsky, M. (2004). Predation risk is an ecological constraint for helper dispersal in a cooperatively breeding child. Proceedings of the Royal Society of London, Series B - Biological Sciences, 271(1555), 2367-2374. http://dx.doi.org/10.1098/rspb.2004.2855

Khan, Q. J. A., Balakrishanan, E., \& Wake, G. C. (2004). Analysis of a predator-prey system with predator switching. Bulletin of Mathematical Biology, 66(1), 109-123.

Khan, Q. J. A., Bhatt, B. S., \& Jaju, R. P. (1998). Switching model with two habitats and a predator involving group defense. Journal of Nonlinear Mathematical Physics, 5, 212. http://dx.doi.org/10.2991/jnmp.1998.5.2.11 